\title{
MacMahon's theorem for a set of permutations with given descent indices and right-maximal records
}

\author{
A. Dzhumadil'daev \\ Institute of Mathematics, Pushkin street 125, Almaty, Kazakhstan \\ askar56@hotmail.com \\ Submitted: Mar 29, 2009; Accepted: Feb 18, 2010; Published: Feb 28, 2010 \\ Mathematics Subject Classification: 05A05, 05A15
}

\begin{abstract}
We show that the major codes and inversion codes are equidistributed over a set of permutations with prescribed descent indices and right-maximal records.
\end{abstract}

\section{Introduction}

Let $[n]=\{1,2, \ldots, n\}$, and let $S_{n}$ be the set of permutations on $[n]$. We will use the single-line notation for a permutation: we write $\sigma=\sigma(1) \sigma(2) \cdots \sigma(n)$ rather than

$$
\sigma=\left(\begin{array}{cccc}
1 & 2 & \cdots & n \\
\sigma(1) & \sigma(2) & \cdots & \sigma(n)
\end{array}\right)
$$

Given $\sigma \in S_{n}$, we say that $i$ is a descent index of $\sigma$ if $\sigma(i)>\sigma(i+1)$. We let $\operatorname{desi}(\sigma)$ stand for the set of all descent indices of $\sigma \in S_{n}$. The sum of descent indices is called the major index of $\sigma$, denoted by maj $(\sigma)$. We say that $(i, j)$ is an inversion pair if $i<j$ and $\sigma(i)>\sigma(j)$. The number of inversion pairs is referred to as the inversion index of $\sigma$, denoted by $\operatorname{inv}(\sigma)$.

Let

$$
E_{n}=\left\{\alpha=\alpha_{1} \ldots \alpha_{n} \mid 0 \leqslant \alpha_{i} \leqslant n-i, i=1, \ldots, n\right\} .
$$

The members of $E_{n}$ are called the coding words. Any bijective function $f: S_{n} \rightarrow E_{n}$ is referred to as coding of permutations. The inversion code is defined as

$$
\text { invcode }: S_{n} \rightarrow E_{n}, \quad \operatorname{invcode}(\sigma)=c_{1} \ldots c_{n},
$$

where $c_{i}$ is the number of all inversion pairs $|\{j>i \mid \sigma(i)>\sigma(j)\}|$.

The major code is defined as

$$
\text { majcode }: S_{n} \rightarrow E_{n}, \quad \text { majcode }(\sigma)=m_{1} \ldots m_{n},
$$


where

$$
m_{i}=\operatorname{maj}\left(\sigma^{(i)}\right)-\operatorname{maj}\left(\sigma^{(i+1)}\right),
$$

and $\sigma^{(i)}$ is the permutation that is obtained from $\sigma$ by deleting all components less than $i$.

The inverse statistics

$$
\text { Imajcode, Iinvcode }: S_{n} \rightarrow E_{n}
$$

are defined by

$$
\operatorname{Imajcode}(\sigma)=\operatorname{majcode}\left(\sigma^{-1}\right), \quad \operatorname{Iinvcode}(\sigma)=\operatorname{invcode}\left(\sigma^{-1}\right) .
$$

For a permutation $\sigma \in S_{n}$, we say that $i \in[n]$ is a right-maximal index of $\sigma$ and that $\sigma(i)$ is a right-maximal value, if $\sigma(i)>\sigma(j)$ whenever $i<j \leqslant n$. We denote by $\mathrm{r}[\max , \mathrm{i}](\sigma)$ the set of all right-maximal indices of $\sigma$, and let $\mathrm{r}[\max , \mathrm{v}](\sigma)$ denote the set of all right-maximal values. Note that any right-maximal index is a descent index. In other words, for every $\sigma \in S_{n}$

$$
\mathrm{r}[\max , \mathrm{i}] \backslash\{n\} \subseteq \operatorname{desi}(\sigma) .
$$

Given a sequence $\alpha$, we denote by $\operatorname{sort}(\alpha)$ the same sequence $\alpha$ but written down in non-increasing order. For example, the permutation

$$
\sigma=\left(\begin{array}{ccccc}
1 & 2 & 3 & 4 & 5 \\
2 & 5 & 3 & 4 & 1
\end{array}\right) \in S_{5}
$$

or in our notations $\sigma=25341$, has the descent indices 2,4 ; the major index $6=2+4$; the inversion index 5; the right-maximal indices $5,4,2$; the right-maximal values $1,4,5$; and $\operatorname{sort}(\alpha)=14352$. If $\sigma=7415236$ then invcode $(\sigma)=6302000, \operatorname{Iinvcode}(\sigma)=2331110$, majcode $(\sigma)=3030010$ and Imajcode $(\sigma)=6012000$.

MacMahon [10], [11] (see also [8], [12], [9] ) has proved that the major indices and the inversion indices of permutations are equidistributed over the set of all permutations,

$$
\left|\left\{\sigma \in S_{n} \mid \operatorname{inv}(\sigma)=k\right\}\right|=\left|\left\{\sigma \in S_{n} \mid \operatorname{maj}(\sigma)=k\right\}\right|, \quad \forall k .
$$

Foata [2] reproved this by constructing an explicit bijection $\phi: C \rightarrow C$, where $C$ is the set of multiset permutations, such that maj $\sigma=\operatorname{inv} \phi \sigma$ for every permutation $\sigma \in C$. In particular, this result holds true for usual permutation groups when $C=S_{n}$. Foata and Shützenberger [4] have established that the major and inversion indices are equidistributed over the set of permutations with prescribed descent indices: For any subset $A \subseteq[n-1]$,

$$
\left|\left\{\sigma \in S_{n} \mid \operatorname{desi}(\sigma)=A, \operatorname{inv}(\sigma)=k\right\}\right|=\left|\left\{\sigma \in S_{n} \mid \operatorname{desi}(\sigma)=A, \operatorname{maj}\left(\sigma^{-1}\right)=k\right\}\right|, \quad \forall k .
$$

It was shown in [4] that desi $\sigma=\operatorname{desi} \phi(\sigma)$. Some further properties of $\phi$ were established in [1]. It was proved in particular that $r[\max , v] \sigma=r[\max , v] \phi(\sigma)$.

Hivert, Novelli, and Thibon [7] have generalized the result of [4] for major codes and inversion codes: For any subset $A \subseteq[n-1]$ and for any non-increasing coding word $\alpha \in E_{n}$,

$$
\begin{aligned}
& \left|\left\{\sigma \in S_{n} \mid \operatorname{desi}(\sigma)=A, \operatorname{sort}\left(\operatorname{maj} \operatorname{code}\left(\sigma^{(-1)}\right)\right)=\alpha\right\}\right| \\
& \quad=\left|\left\{\sigma \in S_{n} \mid \operatorname{desi}(\sigma)=A, \operatorname{sort}(\operatorname{invcode}(\sigma))=\alpha\right\}\right| .
\end{aligned}
$$


In our paper, the result of [7] is improved further: For any subsets $A, B$ such that $B \backslash\{n\} \subseteq A \subseteq[n-1]$ and for any non-increasing coding word $\alpha \in E_{n}$,

$$
\begin{aligned}
& \left|\left\{\sigma \in S_{n} \mid \operatorname{desi}(\sigma)=A, \mathrm{r}[\max , \mathrm{i}](\sigma)=B, \operatorname{sort}\left(\operatorname{majcode}\left(\sigma^{(-1)}\right)\right)=\alpha\right\}\right| \\
& \quad=\left|\left\{\sigma \in S_{n} \mid \operatorname{desi}(\sigma)=A, \mathrm{r}[\max , \mathrm{i}](\sigma)=B, \operatorname{sort}(\operatorname{invcode}(\sigma))=\alpha\right\}\right| .
\end{aligned}
$$

Moreover, the bi-statistics (r[max, v], majcode) and (r[max, v], Iinvcode) are equidistributed in a strong form (it is not necessary to sort out the majcodes and inversion codes): For any $\alpha \in E_{n}$,

$$
\begin{aligned}
& \left|\left\{\sigma \in S_{n} \mid \mathrm{r}[\max , \mathrm{v}](\sigma)=A, \operatorname{maj} \operatorname{code}(\sigma)=\alpha\right\}\right| \\
= & \left|\left\{\sigma \in S_{n} \mid \mathrm{r}[\max , \mathrm{v}](\sigma)=A, \operatorname{invcode}\left(\sigma^{-1}\right)=\alpha\right\}\right| .
\end{aligned}
$$

Let us formulate the results of our paper in terms of generating functions.

Theorem 1.1 The triple statistics (desi, r[max, i], Imajcode) and (desi, r[max, i], invcode) are equidistributed,

$$
\sum_{\sigma \in S_{n}} x_{\operatorname{desi}(\sigma)} y_{\mathrm{r}[\max , \mathrm{i}](\sigma)} z_{\operatorname{Imaj} \operatorname{code}(\sigma)}=\sum_{\sigma \in S_{n}} x_{\operatorname{desi}(\sigma)} y_{\mathrm{r}[\max , \mathrm{i}](\sigma)} z_{\operatorname{invcode}(\sigma)} .
$$

Theorem 1.2 The bi-statistics (r[max, v], majcode) and (r[max, $]$, Iinvcode) are noncommutative equidistributed,

$$
\sum_{\sigma \in S_{n}} \mathbf{x}_{\mathrm{r}[\max , \mathrm{v}](\sigma)} \mathbf{y}_{\operatorname{majcode}(\sigma)}=\sum_{\sigma \in S_{n}} \mathbf{x}_{\mathrm{r}[\max , \mathrm{v}](\sigma)} \mathbf{y}_{\operatorname{Iinvcode}(\sigma)}
$$

Moreover, as commutative polynomials,

$$
\sum_{\sigma \in S_{n}} x_{\mathrm{r}[\max , \mathrm{v}](\sigma)} y_{\operatorname{majcode}(\sigma)}=x_{n} y_{0} \prod_{j=1}^{n-1}\left(y_{0}+y_{1}+\cdots+y_{j-1}+x_{n-j} y_{j}\right) .
$$

Since

$$
\mathrm{r}[\max , \mathrm{i}]\left(\sigma^{-1}\right)=\operatorname{rev}(\mathrm{r}[\max , \mathrm{v}](\sigma)),
$$

these results can be reformulated as follows:

$$
\begin{aligned}
\sum_{\sigma \in S_{n}} x_{\operatorname{desi}\left(\sigma^{-1}\right)} y_{\mathrm{r}[\max , \mathrm{v}](\sigma)} z_{\text {majcode }(\sigma)} & =\sum_{\sigma \in S_{n}} x_{\operatorname{desi}\left(\sigma^{-1}\right)} y_{\mathrm{r}[\max , \mathrm{v}](\sigma)} y_{\operatorname{invcode}(\sigma)} . \\
\sum_{\sigma \in S_{n}} \mathbf{x}_{\mathrm{r}[\max , \mathrm{i}](\sigma)} \mathbf{y}_{\operatorname{majcode}\left(\sigma^{-1}\right)} & =\sum_{\sigma \in S_{n}} \mathbf{x}_{\mathrm{r}[\max , \mathrm{i}](\sigma)} \mathbf{y}_{\operatorname{invcode}(\sigma)} .
\end{aligned}
$$

In fact, [7] contains one more result. They introduce one more code, the so called saillane code, denoted by scode, and proved that the bi-statistics (Idesi, majcode) and (Idesi, scode) are equidistributed as well. An extension of Hivert's result in other directions is given in [6]. 
There exist other kinds of permutation records. These depend on three parameters: direction (right-to-left or left-to-right), extremum (maximum or minimum) and place (index or value). Write down a permutation record briefly as $f[g, h]$, where $f=\mathrm{r}, 1 ; g=$ $\max , \min$; and $h=\mathrm{i}, \mathrm{v}$. Here "r,l" corresponds to "right-to-left, left-to-right"; "max,min" to "maximum, minimum"; and "i,v" to "index, value".

Example. If $\sigma=516423$, then

$$
\begin{gathered}
\mathrm{l}[\min , \mathrm{v}](\sigma)=51,1[\min , \mathrm{i}](\sigma)=12, \mathrm{l}[\max , \mathrm{v}](\sigma)=56,1[\max , \mathrm{i}](\sigma)=13, \\
\mathrm{r}[\min , \mathrm{v}](\sigma)=321, \mathrm{r}[\min , \mathrm{i}](\sigma)=652, \mathrm{r}[\max , \mathrm{v}](\sigma)=346, \mathrm{r}[\max , \mathrm{i}](\sigma)=643 .
\end{gathered}
$$

The natural question appears of whether other kinds of records save equidistribution of major codes and inversion codes. We show that Theorem 1.2 cannot be improved. Changing the major (inversion) code to the saillance code is not possible. Changing the right-maximal records to other kinds of records is not possible either.

Theorem 1.3 Let $f$ be one of the following eight kinds of permutation records on $S_{n}$,

$$
\mathrm{r}[\min , \mathrm{i}], \mathrm{r}[\min , \mathrm{v}], \mathrm{r}[\max , \mathrm{i}], \mathrm{r}[\max , \mathrm{v}], 1[\min , \mathrm{i}], \mathrm{l}[\min , \mathrm{v}], \mathrm{l}[\max , \mathrm{i}], \mathrm{l}[\max , \mathrm{v}] \text {. }
$$

Then the permutation bi-statistics ( $f$, majcode) and ( $f$, Invcode) are equidistributed if and only if $f=\mathrm{r}[\max , \mathrm{v}]$. The bi-statistics ( $f$, majcode $),(f$, scode) are not equidistributed.

More exactly, we establish that, if $f=\mathrm{r}[\mathrm{min}, \mathrm{v}]$ is the right-minimal values record, then

$$
\sum_{\sigma \in S_{n}} x_{f(\sigma)} y_{\text {majcode }(\sigma)}=\sum_{\sigma \in S_{n}} x_{f(\sigma)} y_{\text {invcode }\left(\sigma^{(-1)}\right)}
$$

for $n=2,3,4$ but not for $n=5$. For the other six kinds of records, $f=\mathrm{r}[\max , \mathrm{i}], \mathrm{r}[\min , \mathrm{i}]$, $\mathrm{l}[\max , \mathrm{v}], \mathrm{l}[\max , \mathrm{i}], \mathrm{l}[\min , \mathrm{v}], \mathrm{l}[\min , \mathrm{i}]$ and for the bi-statistics ( $f$, majcode), $(f$, scode), counter-examples appear at $n=3$.

\section{Main Lemmas}

For a coding word $\alpha=\alpha_{1} \ldots \alpha_{n} \in E_{n}$, we say that $i$ is a right-maximal index and $\alpha_{i}$ is a right-maximal value of $\alpha$, if $\alpha_{i}=n-i$.

Example. $\alpha=140200 \Rightarrow \mathrm{r}[\max , \mathrm{i}](\alpha)=642, \mathrm{r}[\max , \mathrm{v}](\alpha)=024$.

Lemma $2.1 \mathrm{r}[\max , \mathrm{i}](\operatorname{invcode}(\sigma))=\mathrm{r}[\max , \mathrm{i}](\sigma)$.

Proof. Let $c=\operatorname{invcode}(\sigma)=c_{1} \ldots c_{n}$. Since $c_{i} \leqslant n-i, c_{i}$ reaches a maximum if and only if $c_{i}=n-i$. Clearly, the condition $c_{i}=n-i$ is equivalent to the condition $\sigma(i)>\sigma(j)$ for every $j=i+1, \ldots, n$. This means that $i$ is a right-maximal index of the coding word $c \in E_{n}$ if and only if $i$ is a right-maximal index of the permutation $\sigma \in S_{n}$. In other words,

$$
c_{i}=n-i \Leftrightarrow i \text { is a right-maximal index of } \sigma \text {. }
$$


Lemma $2.2 \mathrm{r}[\max , \mathrm{i}](\operatorname{maj} \operatorname{code}(\sigma))=\operatorname{rev}(\mathrm{r}[\max , \mathrm{v}](\sigma))$.

Proof. Let $m=$ majcode $(\sigma)$ and

$$
\mathrm{r}[\max , \mathrm{v}](\sigma)=r_{1} \ldots r_{k}
$$

Recall that

$$
1 \leqslant r_{1}<r_{2}<\cdots<r_{k}=n
$$

and $r_{i}$ is greater than any element of $\sigma$ on the right of $r_{i}$.

Let last $\left(\sigma^{(i)}\right)$ be the last element of $\sigma^{(i)}$. We will look for the last elements of the sequence $\sigma^{(1)}, \ldots, \sigma^{(n)}$. Let

$$
\tau=\tau_{1} \ldots \tau_{n}, \quad \tau_{i}=\operatorname{last}\left(\sigma^{(i)}\right)
$$

Note that

$$
\tau=\underbrace{\overbrace{\underbrace{r_{1} \ldots r_{1} \text { times }}_{1}}^{r_{k} \ldots r_{2} \ldots r_{k} \ldots r_{k}}}_{r_{2} \text { times }}
$$

Therefore, $n-i$ is a descent index of $\sigma^{(i)}$ if $i$ is a descent value of the permutation $\sigma$. In other words,

$$
\operatorname{desi}\left(\sigma^{(i)}\right)=\operatorname{desi}\left(\sigma^{(i+1)}\right) \cup\{n-i\}
$$

if and only if $i \in \operatorname{desv}(\sigma)$. So,

$$
m_{i}=n-i \Leftrightarrow i \text { is a right-maximal value of } \sigma \text {. }
$$

Example. Let $\sigma=293785614$. Then

$$
\begin{gathered}
\operatorname{invcode}(\sigma)=171442200, \quad \text { majcode }(\sigma)=032503010 \\
\mathrm{r}[\max , \mathrm{i}](\sigma)=9752, \mathrm{r}[\max , \mathrm{v}](\sigma)=4689
\end{gathered}
$$

We see that

$$
\begin{gathered}
\mathrm{r}[\max , \mathrm{i}](\operatorname{maj} \operatorname{code}(\sigma))=9864=\operatorname{rev}(\mathrm{r}[\max , \mathrm{v}](\sigma)), \\
\mathrm{r}[\max , \mathrm{i}](\operatorname{invcode}(\sigma))=9752=\mathrm{r}[\max , \mathrm{i}](\sigma) .
\end{gathered}
$$

Example. Let $\sigma=86742153$. Then

$$
\sigma=86742153 \Rightarrow \mathrm{r}[\max , \mathrm{v}](\sigma)=3578
$$


and

$\begin{array}{lll}i & \sigma^{(i)} & \tau_{i} \\ 1 & 86742153 & 3 \\ 2 & 8674253 & 3 \\ 3 & 867453 & 3 \\ 4 & 86745 & 5 \\ 5 & 8675 & 5 \\ 6 & 867 & 7 \\ 7 & 87 & 7 \\ 8 & 8 & 8\end{array}$

Therefore, the sequence of last elements is

$$
\tau=33355778
$$

Further,

$$
\begin{array}{lll}
i & \sigma^{(i)} & \operatorname{maj}\left(\sigma^{(i)}\right) \\
1 & 86742153 & 1+3+4+5+7=20 \\
2 & 8674253 & 1+3+4+6=14 \\
3 & 867453 & 1+3+5=9 \\
4 & 86745 & 1+3=4 \\
5 & 8675 & 1+3=4 \\
6 & 867 & 1 \\
7 & 87 & 1 \\
8 & 8 & 0
\end{array}
$$

Thus,

$$
m_{1}=6, m_{2}=5, m_{3}=5, m_{4}=0, m_{5}=3, m_{6}=0, m_{7}=1, m_{8}=0 .
$$

We see that $m=65503010$ and

$$
\mathrm{r}[\max , \mathrm{i}](\operatorname{maj} \operatorname{code}(\sigma))=8753=\operatorname{rev}(\mathrm{r}[\max , \mathrm{v}](\sigma)) .
$$

Lemma $2.3 \operatorname{rev}(\mathrm{r}[\max , \mathrm{i}](\sigma))=\mathrm{r}[\max , \mathrm{v}]\left(\sigma^{-1}\right)$.

Proof. Let $\mathrm{r}[\max , \mathrm{i}](\sigma)=i_{1} \ldots i_{k}$. Then

$$
\sigma\left(i_{k}\right)=n>\sigma\left(i_{k-1}\right)>\cdots>\sigma\left(i_{1}\right), \quad i_{1}=n>i_{2}>\cdots>i_{k} .
$$

Moreover, $\sigma\left(i_{s}\right)>\sigma(j)$ for any $i_{s}<j \leqslant n, s=1, \ldots, k$. Therefore,

$$
\mathrm{r}[\max , \mathrm{v}]\left(\sigma^{-1}\right)=i_{k} i_{k-1} \cdots i_{1} \text {. }
$$

In view of Lemma 2.3, Lemmas 2.1 and 2.2 can be rewritten as

$$
\mathrm{r}[\max , \mathrm{i}](\operatorname{maj} \operatorname{code} \sigma)=\mathrm{r}[\max , \mathrm{i}](\operatorname{Iinvcode}(\sigma))=\operatorname{rev}(\mathrm{r}[\max , \mathrm{v}](\sigma))
$$




\section{Proof of Theorem 1.1}

Let $A=\{a, b, c, \ldots\}$ be an alphabet, $A^{*}$ the set of (non-commutative) words on $A$, and $\epsilon$ the empty word. The shuffle product $w_{1} \sqcup w_{2}$ of two words $w_{1}$ and $w_{2}$ is defined recursively by $w_{1} \sqcup \epsilon=w_{1}, \epsilon \sqcup w_{2}=w_{2}$ and

$$
a u \sqcup b v=a(u \sqcup b v)+b(a u \sqcup v), \quad a, b \in A, u, v \in A^{*} .
$$

For example,

$$
a b \sqcup \sqcup c d=a b c d+a c b d+a c d b+c a b d+c a d b+c d a b .
$$

For a word $w=w_{1} \cdots w_{n}$ over the integers, and $k \in \mathbf{N}$, we denote by $w[k]$ the shifted word

$$
w[k]:=\left(w_{1}+k\right) \cdot\left(w_{2}+k\right) \cdots\left(w_{n}+k\right) .
$$

The shifted shuffle of two permutations $\alpha \in S_{k}$ and $\beta \in S_{l}$ is defined by

$$
\alpha \cup \beta:=a \sqcup(\beta[k]) .
$$

A composition of an integer $n$ is a sequence of positive integers of total sum $n$. The descent set $\operatorname{Des}(I)$ of a composition $I=\left(i_{1}, \ldots, i_{r}\right)$ is the set of partial sums $\left\{i_{1}, i_{1}+\right.$ $\left.i_{2}, \ldots, i_{1}+\cdots+i_{r}\right\}$. Compositions are ordered by $I \leqslant J$ iff $\operatorname{Des}(I) \subseteq \operatorname{Des}(J)$. In this case we say that $I$ is coarser than $J$.

The descent composition $I=C(\sigma)$ of a permutation $\sigma \in S_{n}$ is the composition of $n$ whose descents are exactly the set of descent indices of $\sigma$,

$$
\operatorname{Des}(I)=\operatorname{desi}(\sigma)
$$

If $I=\left(i_{1}, \ldots, i_{r}\right)$ is a composition of $n$, then we let $D_{\leqslant I}$ be the sum of all permutations each having descent composition coarser than $I$. Then

$$
D_{\leqslant I}=\left(i d_{i_{1}} \cup i d_{i_{2}} \cup \cdots \cup i d_{i_{r}}\right)^{\vee} .
$$

Here ${ }^{\vee}$ is the linear involution sending each permutation to its inverse and $i d_{s}=12 \cdots s$ is the identity permutation of size $s$. The sum of all permutations whose descent composition is $I$ will be denoted by $D_{I}$.

For example, the descent composition of the permutation $\sigma=52413$ is $I=(1,2,2)$ and

$$
\begin{aligned}
D_{\leqslant I}=\{ & 12345,21345,31245,41235,51234,12435,21435,31425, \\
& 41325,51324,12534,21534,31524,41523,51423,13425, \\
& 23415,32415,42315,52314,13524,23514,32514,42513, \\
& 52413,14523,24513,34512,43512,53412\}, \\
D_{I}=\{ & 21435,21534,31425,31524,32415,32514,41325,41523, \\
& 42315,42513,43512,51324,51423,52314,52413,53412\} .
\end{aligned}
$$


Recall that the algebra Sym of noncommutative symmetric functions is the free associative algebra, on the symbol set $S_{n}$, whose basis is given by $S^{I}=S_{i_{1}} \cdots S_{i_{n}}$ for all compositions $I=\left(i_{1}, \ldots, i_{r}\right)[5]$. When $A$ is an ordered alphabet, $S_{n}(A)$ can be realized as the sum of all nondecreasing words in $A^{n}$. The commutative image of Sym is the algebra of symmetric functions. The $S_{n}$ are mapped to the usual complete homogeneous functions $h_{n}$.

If $I=\left(i_{1}, \ldots, i_{r}\right)$ is a composition of $n$ and $Y_{n}=\left\{y_{0}, y_{1}, \ldots, y_{n}\right\}, Z_{s}=\left\{z_{0}, z_{1}, \ldots, z_{s}\right\}$, then we denote by $\tilde{h}_{k}\left(Y_{n}, Z_{s}\right)$ the polynomial

$$
\tilde{h}_{k}\left(Y_{n}, Z_{s}\right)=\sum_{0 \leqslant i_{0} \leqslant i_{1} \leqslant \cdots \leqslant i_{k-1}<s} z_{i_{0}} z_{i_{1}} \cdots z_{i_{k-1}}+y_{n-s} \sum_{0 \leqslant i_{0} \leqslant i_{1} \leqslant \cdots \leqslant i_{k-2} \leqslant i_{k-1}=s} z_{i_{0}} z_{i_{1}} \cdots z_{i_{k-2}} z_{s} .
$$

For example,

$$
\tilde{h}_{3}\left(Y_{7}, Z_{2}\right)=z_{0}^{3}+z_{0}^{2} z_{1}+z_{0} z_{1}^{2}+z_{1}^{3}+y_{5}\left(z_{0}^{2} z_{2}+z_{0} z_{1} z_{2}+z_{1}^{2} z_{2}+z_{0} z_{2}^{2}+z_{1} z_{2}^{2}+z_{2}^{3}\right) .
$$

Lemma 3.1 Let $I=\left(i_{1}, \ldots, i_{n}\right)$ be a composition of $n$. Then

$$
\begin{aligned}
\sum_{\sigma \in i d_{i_{1}} \cup \cdots \cup i d_{i_{r}}} y_{\mathrm{r}}[\max , \mathrm{v}](\sigma) & z_{\text {Iinvcode }(\sigma)} \\
& =\tilde{h}_{i_{1}}\left(Y_{n}, Z_{i_{2}+\cdots+i_{r}}\right) \tilde{h}_{i_{2}}\left(Y_{n}, Z_{i_{3}+\cdots+i_{r}}\right) \cdots \tilde{h}_{i_{r-1}}\left(Y_{n}, Z_{i_{r}}\right) \tilde{h}_{i_{r}}\left(Y_{n}, Z_{0}\right) .
\end{aligned}
$$

Proof repeats the proof of Theorem 5.1 of [7]. We use the induction on the number of parts of $I$. The statement is obvious for $r=1$. Suppose that our statement is true for the composition $\left(i_{2}, \ldots, i_{r}\right)$. Let us prove it for $I$.

Let $\sigma$ be an element of $i d_{i_{2}} \cup \cdots \cup i d_{i_{r}}$ and let $\gamma$ be any element in $i d_{i_{1}} \cup \sigma$. Then $I c_{i_{1}+k}(\gamma)=I c_{k}(\sigma)$ for all $k$, where $I c_{j}(\alpha)$ are the components of the inversion code of a permutation $\alpha$. Moreover, the sequence $I c_{k}(\gamma)$ for $k \in\left[1, i_{1}\right]$ is nondecreasing, since $1, \ldots, i_{1}$ are in this order in $\gamma$, and it is bounded by the number of letters of $\sigma$; i.e., $i_{2}+\cdots+i_{r}$. Hence, the maximum of $I c_{k}(\gamma)$ is $i_{2}+\cdots+i_{r}$, and, by relation (2),

$$
\mathrm{r}[\max , \mathrm{v}] \gamma=n-i_{2}-\cdots-i_{r} .
$$

The invcode is a bijection. Therefore, no two words $\gamma$ may have the same code. In particular, the first $i_{1}$ values will be different if $\gamma$ runs through the elements of $i d_{i_{1}} \cup \sigma$. On the other hand, the number of elements in $i d_{i_{1}} \cup \sigma$ is equal to the number of nondecreasing sequences in $\left[0, i_{2}+\cdots+i_{r}\right]$. Hence all sequences appear, and

$$
\sum_{\gamma \in i d_{i_{1}} \cup \sigma} y_{\mathrm{r}[\max , \mathrm{v}](\gamma)} z_{\operatorname{Iinvcode}(\gamma)}=\tilde{h}_{i_{1}}\left(Y_{n}, Z_{i_{2}+\cdots+i_{r}}\right) y_{\mathrm{r}[\max , \mathrm{v}](\sigma)} z_{\operatorname{Iinvcode}(\sigma)} .
$$

Lemma 3.2 Let $I=\left(i_{1}, \ldots, i_{n}\right)$ be a composition of $n$. Then

$$
\begin{aligned}
& \sum_{\sigma \in i d_{i_{1}} \cup \cdots \cup i d_{i_{r}}} y_{\mathrm{r}[\max , \mathrm{v}](\sigma)} z_{\text {majcode }(\sigma)} \\
& \quad=\tilde{h}_{i_{1}}\left(Y_{n}, Z_{i_{2}+\cdots+i_{r}}\right) \tilde{h}_{i_{2}}\left(Y_{n}, Z_{i_{3}+\cdots+i_{r}}\right) \cdots \tilde{h}_{i_{r-1}}\left(Y_{n}, Z_{i_{r}}\right) \tilde{h}_{i_{r}}\left(Y_{n}, Z_{0}\right)
\end{aligned}
$$


Proof of Lemma 3.2 repeats the proof of relation (68) of [7]. It follows from four lemmas of [7], namely Lemmas 6.2, 6.3, 6.4 and 6.5. Recall that Lemma 6.5 of [7] states the following.

Let $\beta \in S_{n}$ and $k$ be an integer. The set of sorted $k$ first components of the majcodes of the elements in $i d_{k} \cup \beta$ is the set of all sequences $\left(0 \leqslant j_{1} \leqslant j_{2} \leqslant \cdots \leqslant j_{k} \leqslant n\right)$. In particular, we have

$$
\sum_{\sigma \in i d_{k} \cup \beta} x_{\text {majcode }(\sigma)}=h_{k}\left(X_{n}\right) x_{\text {majcode }(\beta)} .
$$

We are to specify this Lemma as follows:

$$
\sum_{\sigma \in i d_{i_{1}} \cup \cdots \cup i d_{i_{r}}} y_{\mathrm{r}[\max , \mathrm{v}](\sigma)} z_{\operatorname{majcode}(\sigma)}=\tilde{h}_{i_{1}}\left(Y_{n}, Z_{n-i_{1}}\right) \sum_{\beta \in i d_{i_{2}} \cup \cdots \cup i d_{i_{r}}} y_{\mathrm{r}[\max , \mathrm{v}](\beta)} z_{\operatorname{majcode}(\beta)}
$$

Let us prove this specification. For any $\beta \in i d_{i_{2}} \cup \cdots \cup i d_{i_{r}}$ the set of the sorted $i_{1}$ first components of the majcodes of the elements in $i d_{i_{1}} \cup \beta$ is the set of all sequences $0 \leqslant j_{1} \leqslant j_{2} \leqslant \cdots \leqslant j_{i_{1}} \leqslant i_{2}+\cdots+i_{r}$. Therefore, the maximum in the $i_{1}$ first components of the majcodes of the elements in $i d_{i_{1}} \cup \beta$ is $i_{2}+\cdots+i_{r}=n-i_{1}$. By (2) this means that the right-maximal record values of the elements in $i d_{i_{1}} \cup \beta$ appear iff the majcodes of these elements reach the maximal value $i_{2}+\cdots+i_{r}$.

Proof of Theorem 1.1. The claim follows from Lemmas 3.1 and 3.2.

As in [7], Theorem 1.1 (more exactly Lemma 3.1) implies the following statement.

Corollary 3.3 The commutative generating series for the bi-statistic (r[max, i], invcode) on a descent class is given by the following determinant

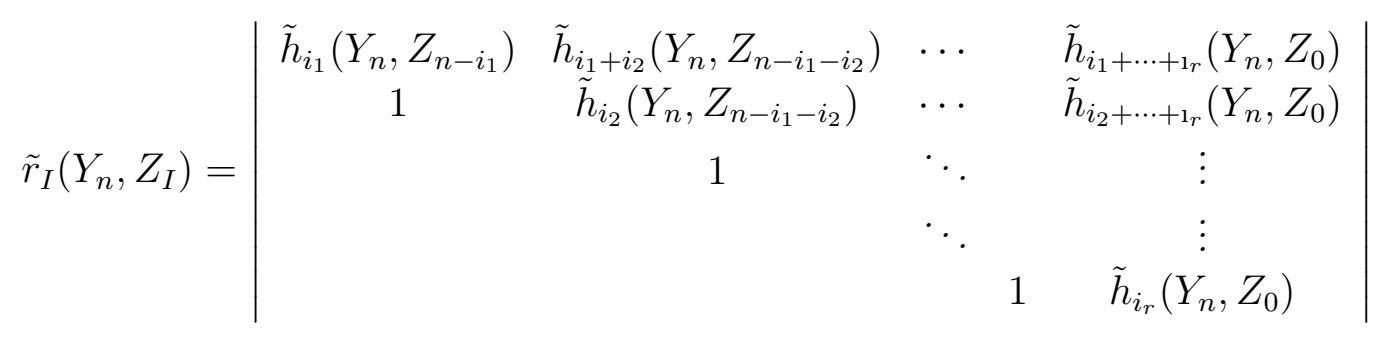

\section{Proof of Theorem 1.2}

Since major codes and inversion codes are bijective maps, we have the inverse maps

$$
\text { majcode }^{-1}: E_{n} \rightarrow S_{n}, \text { Iinvcode }^{-1}: E_{n} \rightarrow S_{n} .
$$

By Lemmas 2.1, 2.2 and 2.3,

$$
\mathrm{r}[\max , \mathrm{v}]\left(\operatorname{majcode}^{-1}(\alpha)\right)=\mathrm{r}[\max , \mathrm{v}]\left(\operatorname{Iinvcode}^{-1}(\alpha) .\right.
$$


Therefore,

$$
\begin{aligned}
\sum_{\sigma \in S_{n}} \mathbf{x}_{\mathrm{r}[\max , \mathrm{v}](\sigma)} \mathbf{y}_{\text {majcode }(\sigma)} & =\sum_{\alpha \in E_{n}} \mathbf{x}_{\mathrm{r}[\max , \mathrm{v}]\left(\text { majcode }^{-1}(\alpha)\right)} \mathbf{y}_{\alpha} \\
& =\sum_{\alpha \in E_{n}} \mathbf{x}_{\mathrm{r}[\max , \mathrm{v}]\left(\operatorname{Iinvcode}^{-1}(\alpha)\right)} \mathbf{y}_{\alpha} \\
& =\sum_{\sigma \in S_{n}} \mathbf{x}_{\mathrm{r}[\max , \mathrm{v}](\sigma)} \mathbf{y}_{\operatorname{Iinvcode}(\sigma)} .
\end{aligned}
$$

Suppose now that the variables $x_{1}, \ldots, x_{n}, y_{1}, \ldots, y_{n}$ are commutative. For a coding word $c=c_{1} \ldots c_{n} \in E_{n}$, set

$$
\bar{c}_{i}= \begin{cases}i & \text { if } c_{i}=n-i \\ 0 & \text { otherwise }\end{cases}
$$

If $c=\operatorname{invcode}(\sigma)$ for some $\sigma \in S_{n}$, then by Lemma $2.1 \bar{c}_{i}=i$ if and only if $i$ is a right-maximal index of $\sigma$. Therefore,

$$
\begin{aligned}
\sum_{\sigma \in S_{n}} x_{\mathrm{r}[\max , \mathrm{i}](\sigma)} y_{\text {invcode }(\sigma)} & =\sum_{c \in E_{n}} x_{\bar{c}_{1}} \cdots x_{\bar{c}_{n}} y_{c_{1}} \cdots y_{c_{n}} \\
& =\sum_{c_{1}=0}^{n-1} \sum_{c_{2}=0}^{n-2} \cdots \sum_{c_{n}=0}^{0} x_{\bar{c}_{1}} \cdots x_{\bar{c}_{n}} y_{c_{1}} \cdots y_{c_{n}} \\
& =\sum_{c_{1}=0}^{n-1} x_{\bar{c}_{1}} y_{c_{1}} \sum_{c_{2}=0}^{n-2} x_{\bar{c}_{2}} y_{c_{2}} \cdots \sum_{c_{n}=0}^{0} x_{\bar{c}_{n}} y_{c_{n}} \\
& =\left(x_{1} y_{n-1}+\sum_{c_{1}=0}^{n-2} x_{\bar{c}_{1}} y_{c_{1}}\right)\left(x_{2} y_{n-2}+\sum_{c_{2}=0}^{n-3} x_{\bar{c}_{2}} y_{c_{2}}\right) \cdots\left(x_{n} y_{0}\right) \\
& =\left(x_{1} y_{n-1}+\sum_{c_{1}=0}^{n-2} y_{c_{1}}\right)\left(x_{2} y_{n-2}+\sum_{c_{2}=0}^{n-3} y_{c_{2}}\right) \cdots\left(x_{n} y_{0}\right) \\
& =x_{n} y_{0}\left(y_{0}+x_{n-1} y_{1}\right) \cdots\left(y_{0}+y_{1}+\cdots+y_{n-2}+x_{1} y_{n-1}\right) .
\end{aligned}
$$

Similar arguments apply to majcodes. If $m=\operatorname{majcode}(\sigma)$ for some $\sigma \in S_{n}$, then by Lemma $2.2 \bar{m}_{i}=i$ if and only if $i$ is a right-maximal value of $\sigma$. Therefore,

$$
\begin{aligned}
\sum_{\sigma \in S_{n}} x_{r[\max , v](\sigma)} y_{\text {majcode }(\sigma)} & =\sum_{m \in E_{n}} x_{\bar{m}_{1}} \cdots x_{\bar{m}_{n}} y_{m_{1}} \cdots y_{m_{n}} \\
& =\sum_{m_{1}=0}^{n-1} \sum_{m_{2}=0}^{n-2} \cdots \sum_{m_{n}=0}^{0} x_{\bar{m}_{1}} \cdots x_{\bar{m}_{n}} y_{m_{1}} \cdots y_{m_{n}} \\
& =\sum_{m_{1}=0}^{n-1} x_{\bar{m}_{1}} y_{m_{1}} \sum_{m_{2}=0}^{n-2} x_{\bar{m}_{2}} y_{m_{2}} \cdots \sum_{m_{n}=0}^{0} x_{\bar{m}_{n}} y_{m_{n}}
\end{aligned}
$$




$$
\begin{aligned}
& =\left(x_{1} y_{n-1}+\sum_{m_{1}=0}^{n-2} x_{\bar{m}_{1}} y_{m_{1}}\right)\left(x_{2} y_{n-2}+\sum_{m_{2}=0}^{n-3} x_{\bar{m}_{2}} y_{m_{2}}\right) \cdots\left(x_{n} y_{0}\right) \\
& =\left(x_{1} y_{n-1}+\sum_{m_{1}=0}^{n-2} y_{m_{1}}\right)\left(x_{2} y_{n-2}+\sum_{m_{2}=0}^{n-3} y_{m_{2}}\right) \cdots\left(x_{n} y_{0}\right) \\
& =x_{n} y_{0}\left(y_{0}+x_{n-1} y_{1}\right) \cdots\left(y_{0}+y_{1}+\cdots+y_{n-2}+x_{1} y_{n-1}\right) .
\end{aligned}
$$

So, the bi-statistics (r[max, i], invcode) and (r[max, v], majcode) are equidistributed and the generating functions are given by (1).

\section{$5 \quad$ Proof of Theorem 1.3}

Theorem 1.3 can be reformulated as follows:

$$
\sum_{\sigma \in S_{n}} x_{\mathrm{r}[\max , \mathrm{v}](\sigma)} y_{\text {majcode }(\sigma)}=\sum_{\sigma \in S_{n}} x_{\mathrm{r}[\max , \mathrm{v}](\sigma)} y_{\operatorname{linvcode}(\sigma)} .
$$

In this section we show that changing right-maximal records to other kinds of records is not possible here. We prove the following result:

The permutation statistics ( $f$, majcode) and $(f$, Iinvcode) are not equidistributed if $f=l[a, b], a=\min , \max , b=i, v$ or $f=r[\max , i], r[\min , i], r[\min , v]$.

Consider the test functions

$$
\begin{gathered}
\text { test }_{1}(k, f)=\sum_{\sigma \in S_{k}} x_{f(\sigma)} y_{\text {invcode }\left(\sigma^{-1}\right)}, \quad \text { test }_{2}(k, f)=\sum_{\sigma \in S_{k}} x_{f(\sigma)} y_{\text {majcode }(\sigma)}, \\
\text { test }(k, f)=\text { test }_{1}(k, f)-\text { test }_{2}(k, f) .
\end{gathered}
$$

To simplify calculations, set $x_{0}=y_{0}=1$.

For $n=3$, the following relations hold:

$$
\begin{aligned}
& \text { test }_{1}(3, \mathrm{l}[\mathrm{min}, \mathrm{v}])=x_{1}+x_{1} y_{1}+x_{1} x_{2} y_{1}+x_{1} x_{3} y_{1}^{2}+x_{1} x_{2} y_{2}+x_{1} x_{2} x_{3} y_{1} y_{2}, \\
& \text { test }_{2}(3, \mathrm{l}[\mathrm{min}, \mathrm{v}])=x_{1}+x_{1} x_{2} y_{1}+x_{1} x_{3} y_{1}+x_{1} y_{1}^{2}+x_{1} x_{2} y_{2}+x_{1} x_{2} x_{3} y_{1} y_{2}, \\
& \operatorname{test}(3, \mathrm{l}[\mathrm{min}, \mathrm{v}])=x_{1} y_{1}\left(-1+x_{3}\right)\left(-1+y_{1}\right), \\
& \text { test }_{1}(3, \mathrm{l}[\mathrm{min}, \mathrm{i}])=x_{1}+x_{1} y_{1}+x_{1} x_{2} y_{1}+x_{1} x_{2} y_{1}^{2}+x_{1} x_{3} y_{2}+x_{1} x_{2} x_{3} y_{1} y_{2}, \\
& \operatorname{test}_{2}(3, \mathrm{l}[\min , \mathrm{i}])=x_{1}+2 x_{1} x_{2} y_{1}+x_{1} y_{1}^{2}+x_{1} x_{3} y_{2}+x_{1} x_{2} x_{3} y_{1} y_{2}, \\
& \operatorname{test}(3, \mathrm{l}[\min , \mathrm{i}])=x_{1} y_{1}\left(-1+x_{2}\right)\left(-1+y_{1}\right), \\
& \text { test }_{1}(3, \mathrm{l}[\max , \mathrm{v}])=x_{1} x_{2} x_{3}+x_{1} x_{3} y_{1}+x_{2} x_{3} y_{1}+x_{3} y_{1}^{2}+x_{2} x_{3} y_{2}+x_{3} y_{1} y_{2}, \\
& \text { test }_{2}(3, \mathrm{l}[\max , \mathrm{v}])=x_{1} x_{2} x_{3}+x_{3} y_{1}+x_{2} x_{3} y_{1}+x_{1} x_{3} y_{1}^{2}+x_{2} x_{3} y_{2}+x_{3} y_{1} y_{2}, \\
& \operatorname{test}(3,1[\max , \mathrm{v}])=-y_{1}\left(-1+x_{1}\right) x_{3}\left(-1+y_{1}\right),
\end{aligned}
$$




$$
\begin{aligned}
& \text { test }_{1}(3, \mathrm{l}[\max , \mathrm{i}])=x_{1} x_{2} x_{3}+x_{1} x_{2} y_{1}+x_{1} x_{3} y_{1}+x_{1} y_{1}^{2}+x_{1} x_{2} y_{2}+x_{1} y_{1} y_{2} \text {, } \\
& \text { test }_{2}(3,1[\max , \mathrm{v}])=x_{1} x_{2} x_{3}+x_{1} y_{1}+x_{1} x_{3} y_{1}+x_{1} x_{2} y_{1}^{2}+x_{1} x_{2} y_{2}+x_{1} y_{1} y_{2} \text {, } \\
& \operatorname{test}(3, \mathrm{l}[\max , \mathrm{v}])=-x_{1} y_{1}\left(-1+x_{2}\right)\left(-1+y_{1}\right) \text {, } \\
& \text { test }_{1}(3, \mathrm{r}[\max , \mathrm{i}])=x_{3}+x_{3} y_{1}+x_{2} x_{3} y_{1}+x_{1} x_{3} y_{1}^{2}+x_{2} x_{3} y_{2}+x_{1} x_{2} x_{3} y_{1} y_{2} \text {, } \\
& \text { test }_{2}(3, \mathrm{r}[\max , \mathrm{i}])=x_{3}+x_{3} y_{1}+x_{1} x_{3} y_{1}+x_{2} x_{3} y_{1}^{2}+x_{2} x_{3} y_{2}+x_{1} x_{2} x_{3} y_{1} y_{2} \text {, } \\
& \operatorname{test}(3, \mathrm{r}[\max , \mathrm{i}])=y_{1}\left(x_{1}-x_{2}\right) x_{3}\left(-1+y_{1}\right) \text {, } \\
& \text { test }_{1}(3, \mathrm{r}[\mathrm{min}, \mathrm{v}])=x_{1} x_{2} x_{3}+x_{1} x_{2} y_{1}+x_{1} x_{3} y_{1}+x_{1} x_{2} y_{1}^{2}+x_{1} y_{2}+x_{1} y_{1} y_{2} \text {, } \\
& \text { test }_{2}(3, \mathrm{r}[\mathrm{min}, \mathrm{v}])=x_{1} x_{2} x_{3}+x_{1} x_{2} y_{1}+x_{1} x_{3} y_{1}+x_{1} x_{2} y_{1}^{2}+x_{1} y_{2}+x_{1} y_{1} y_{2} \text {, } \\
& \operatorname{test}(3, \mathrm{r}[\min , \mathrm{v}])=0 \text {, } \\
& \text { test }_{1}(3, \mathrm{r}[\mathrm{min}, \mathrm{i}])=x_{1} x_{2} x_{3}+x_{1} x_{3} y_{1}+x_{2} x_{3} y_{1}+x_{2} x_{3} y_{1}^{2}+x_{3} y_{2}+x_{3} y_{1} y_{2} \text {, } \\
& \text { test }_{2}(3, \mathrm{r}[\mathrm{min}, \mathrm{i}])=x_{1} x_{2} x_{3}+2 x_{2} x_{3} y_{1}+x_{1} x_{3} y_{1}^{2}+x_{3} y_{2}+x_{3} y_{1} y_{2} \text {, } \\
& \operatorname{test}(3, \mathrm{r}[\min , \mathrm{i}])=-y_{1}\left(x_{1}-x_{2}\right) x_{3}\left(-1+y_{1}\right) \text {. }
\end{aligned}
$$

So, in the six cases $f=1[\min , \mathrm{i}], \mathrm{l}[\min , \mathrm{v}], \mathrm{l}[\max , \mathrm{i}], \mathrm{l}[\max , \mathrm{v}], \mathrm{r}[\min , \mathrm{i}], \mathrm{r}[\max , \mathrm{i}]$ counterexamples appear at $n=3$.

For $f=\mathrm{r}[\min , \mathrm{v}]$ and $n=4$, no counter-examples exist,

$$
\begin{aligned}
\text { test }_{1}(4, \mathrm{r}[\mathrm{min}, \mathrm{v}])=x_{1} & x_{2} x_{3} x_{4}+x_{1} x_{2} x_{3} y_{1}+x_{1} x_{2} x_{4} y_{1}+x_{1} x_{3} x_{4} y_{1}+x_{1} x_{3} y_{1}^{2} \\
& +x_{1} x_{2} x_{3} y_{1}^{2}+x_{1} x_{2} x_{4} y_{1}^{2}+x_{1} x_{2} x_{3} y_{1}^{3}+x_{1} x_{2} y_{2} \\
& +x_{1} x_{4} y_{2}+2 x_{1} x_{2} y_{1} y_{2}+x_{1} x_{3} y_{1} y_{2}+x_{1} x_{4} y_{1} y_{2} \\
& +x_{1} x_{2} y_{1}^{2} y_{2}+x_{1} x_{3} y_{1}^{2} y_{2}+x_{1} x_{2} y_{2}^{2}+x_{1} x_{2} y_{1} y_{2}^{2} \\
& +x_{1} y_{3}+2 x_{1} y_{1} y_{3}+x_{1} y_{1}^{2} y_{3}+x_{1} y_{2} y_{3}+x_{1} y_{1} y_{2} y_{3}, \\
\text { test }_{1}(4, \mathrm{r}[\min , \mathrm{v}])=\text { test }_{2}(4, \mathrm{r}[\min , \mathrm{v}]), & \\
\text { test }(4, \mathrm{r}[\mathrm{min}, \mathrm{v}]) & =\text { test }_{1}(4, \mathrm{r}[\text { min, } \mathrm{v}])-\text { test }_{2}(4, \mathrm{r}[\mathrm{min}, \mathrm{v}])=0 .
\end{aligned}
$$

A counter-example for $f=\mathrm{r}[\mathrm{min}, \mathrm{v}]$ appears at $n=5$. Let us prove this.

Let

$$
\begin{gathered}
M=\left\{\sigma \in S_{5} \mid \mathrm{r}[\min , \mathrm{v}](\sigma)=431\right\} \\
M_{1}=\left\{\sigma^{-1} \in S_{5} \mid \mathrm{r}[\min , \mathrm{v}](\sigma)=431\right\} .
\end{gathered}
$$

Note that

$$
\begin{aligned}
& M=\{21354,21534,25134,52134\} \\
& M_{1}=\{21354,21453,31452,32451\} .
\end{aligned}
$$

Let

$$
\begin{aligned}
& \operatorname{majcode}(M)=\{\operatorname{majcode}(\sigma) \mid \sigma \in M\}, \\
& \operatorname{invcode}\left(M_{1}\right)=\left\{\operatorname{invcode}(\sigma) \mid \sigma \in M_{1}\right\} .
\end{aligned}
$$


Then

$$
\begin{aligned}
& \operatorname{majcode}(M)=\{21110,21010,01010,20010\}, \\
& \operatorname{invcode}\left(M_{1}\right)=\{10010,10110,20110,21110\} .
\end{aligned}
$$

Hence

$$
20010 \in \operatorname{majcode}(M), \quad 20010 \notin \operatorname{invcode}\left(M_{1}\right) .
$$

Moreover, there exists exactly one permutation $\sigma \in S_{5}$ such that

$$
\mathrm{r}[\min , \mathrm{v}](\sigma)=431, \operatorname{maj} \operatorname{code}(\sigma)=20010,
$$

(namely, $\sigma=52134$ ), but there is no permutation $\sigma \in S_{5}$ with the properties

$$
\mathrm{r}[\min , \mathrm{v}](\sigma)=431, \quad y_{\operatorname{invcode}\left(\sigma^{-1}\right)}=y_{1} y_{2} .
$$

So, we have established that the sum $\sum_{\sigma \in S_{5}} x_{\mathrm{r}[\min , \mathrm{v}](\sigma)} y_{\text {majcode }(\sigma)}$ contains the member $x_{1} x_{3} x_{4} y_{1} y_{2}$ with coefficient 1 , whereas the sum $\sum_{\sigma \in S_{5}} x_{\mathrm{r}[\min , \mathrm{v}](\sigma)} y_{\text {invcode }\left(\sigma^{-1}\right)}$ does not; a contradiction.

Similarly, one can check that test $(3, f) \neq 0$ for a test function defined by

$$
\operatorname{test}(k, f)=\sum_{\sigma \in S_{k}} x_{f(\sigma)} y_{\operatorname{scode}(\sigma)}-\sum_{\sigma \in S_{k}} x_{f(\sigma)} y_{\text {majcode }(\sigma)}
$$

Remark. We say that two triple statistics $(f, g, h)$ and $\left(f_{1}, g_{1}, h_{1}\right)$ are equidistributed, and write $(f, g, h) \sim\left(f_{1}, g_{1}, h_{1}\right)$, if their multi-variable generating functions are equal,

$$
\sum_{\sigma \in S_{n}} x_{f(\sigma)} y_{g(\sigma)} z_{h(\sigma)}=\sum_{\sigma \in S_{n}} x_{f_{1}(\sigma)} y_{g_{1}(\sigma)} z_{h_{1}(\sigma)}
$$

Let $\operatorname{Idesi}(\sigma)=\operatorname{desi}\left(\sigma^{-1}\right)$. One can show that the following triple statistics are equidistributed in a weaker form:

(Idesi, $1[\max , \mathrm{v}]$, Iinvcode $) \sim($ Idesi, $1[\max , \mathrm{v}]$, majcode $)$

if $y_{i}=1, i<n-1$,

(Idesi, l[min, v], Iinvcode $) \sim($ Idesi, l[min, v], majcode $)$

if $y_{i}=1, i>2$,

(Idesi, r [min, v], Iinvcode $) \sim($ Idesi, $r[$ min, v], majcode $)$

if $y_{i}=1,2<i<n-1$,

(Idesi, l[min, v], scode $) \sim($ Idesi, l[min, v], majcode $)$

if $y_{i}=1, i>2$,

(Idesi, r $[$ min, v], scode $) \sim($ Idesi, $r[\min , \mathrm{v}]$, majcode $)$ 
if $y_{i}=1, i>2$,

(Idesi, $1[\max , \mathrm{v}]$, scode $) \sim($ Idesi, $\mathrm{l}[\max , \mathrm{v}]$, majcode $)$

if $y_{i}=1, i<n-1$,

(Idesi, $\mathrm{r}[\max , \mathrm{v}]$, scode $) \sim($ Idesi, $\mathrm{r}[\max , \mathrm{v}]$, majcode $)$

if $y_{i}=1, i<n-1$.

\section{Acknowledgments}

I am grateful to N. Bakhytjan and A. Jumadildayeva for assistance in making calculations, and to the anonymous referee for essential remarks.

\section{References}

[1] A. Björner, M.L. Wachs, Permutation Statsitics and Linear extensions of posets, J. Combin. Theory, Ser. A, 58(1991),85-114.

[2] D. Foata, On the Netto inversion number of a sequence, Proc. AMS., 19(1968), 236240.

[3] D. Foata, G.-N. Han, Un nouvelle transormation pour les statistiques Eulermahoniennes ensemblistes, Moscow Math. J., 4(2004), 131-152.

[4] D. Foata, M.P. Schützenberger, Major index and inversion number of permutations, Math. Nachr., 83(1970), 143-159.

[5] I.M. Gelfand, D. Krob, A. Lascoux, B. Leclerc, V.S. Retakh, Y.-L. Thibon, Noncommutative symmetric functions, Adv. in Math., 112(1995), 218-348.

[6] G.-N. Han, Euler-Mahonian triple set-valued statistics on permutations, Europ. J. Comb., 29(2008), 568-580.

[7] F.Hivert, J-C. Novelli, J-Y. Thibon, Multivariate generalizations of the FoataSchützenberger equidistribution, Fourth Colloquium on Mathematics and Computer Science, Discrete Mathematics and Theoretical Computer Science, proc. AG, 2006, 289-300.

[8] D. Knuth, The Art of Computer Programming, v.3, Addison-Wesley, 1998.

[9] M. Lothaire, Combinatorics on words, Addison-Wesley, London (1983), Encyc. Math. Appl., 17.

[10] P.A. MacMahon, The indices of permutations and derivation therefrom of functions of a single variable associated with the permutations of any assemblage of objects, Amer. J. Math., 35(1913), 281-322.

[11] P. A. MacMahon, Two applictions of general theorems in combinatory analysis, Proc. Londin Math. Soc., 15(1916), 314-321.

[12] R. Stanley, Enumerative combinatorics, v.1, Waldsworth, Inc.California, 1986. 